\title{
CIENCIA Y TECNOLOGÍA PARA UN DESARROLLO PERDURABLE
}

\author{
SCIENCE AND TECHNOLOGY FOR LASTING DEVELOPMENT
}

CIÊNCIA E TECNOLOGIA PARA UM DESENVOLVIMENTO DURADOURO

\section{Pedro César Cantú-Martínez ${ }^{1}$}

\section{Resumen}

La ciencia y la tecnología es un saber transcendental que genera un auge económico en la sociedad. Su aportación al desarrollo sustentable consiste en la generación de capacidades sociales que permiten mostrar la autonomía socioeconómica para la resolución de los problemas más apremiantes. En el presente manuscrito se hace una exploración de este rol para enfrentar los desafíos actuales en un marco de carácter regional para América Latina y el Caribe.

Palabras clave: ciencia; tecnología; desarrollo; sustentabilidad

\begin{abstract}
Knowledge of science and technology is critical to generate economic development. Its contribution to sustainable development lies in the generation of social capabilities that make it possible to achieve socioeconomic autonomy for solving critical problems. The present paper explores the role of this knowledge in confronting the current challenges within a regional framework for Latin America and the Caribbean.
\end{abstract}

Key words: science; technology; development; sustainability

Doi: http://dx.doi.org/10.15359/eys.24-55.7

Fecha de recepción: 28-09-2018. Fechas de reenvíos: 20-11-2018, 23-01-2019, 02-04-2019, 08-05-2019. Aceptado el 04-06-2019. Publicado el 20-06-2019.

${ }^{1}$ Doctor en Ciencias, Profesor Investigador de la Universidad Autónoma de Nuevo León, adscrito a la Facultad de Ciencias Biológicas, San Nicolás de los Garza, N.L., México. Correo Electrónico: cantup@hotmail.com. ORCID: https://orcid.org/0000-0001-8924-5343 


\begin{abstract}
Resumo
Ciência e tecnologia é um conhecimento transcendental que gera um ápice econômico na sociedade. Sua contribuição para o desenvolvimento sustentável consiste na geração de capacidades sociais que permitem mostrar a autonomia socioeconômica para a resolução dos problemas mais prementes. Este manuscrito explora esse papel para enfrentar os atuais desafios em um âmbito de caráter regional para a América Latina e o Caribe.
\end{abstract}

Palavras-chaves: ciência; tecnologia; desenvolvimento; sustentabilidade

\title{
1. Introducción
}

La ciencia y tecnología (CyT) son elementos clave del bienestar sustentable en las sociedades modernas, ya que enriquecen el patrimonio cultural de las naciones y estimulan la capacidad para innovar (Cantú Martínez, 2017); en este aspecto la Organización de las Naciones Unidas para la Educación, la Ciencia y la Cultura (UNESCO) (2005, p. 175) agrega que el conocimiento tecnocientífico es "fuente de autonomía y de creación de capacidades, [y] puede ser un instrumento decisivo del desarrollo". Reconoce que la ciencia y tecnología, deben de utilizarse para satisfacer las necesidades básicas del ser humano como son: la salud, el abastecimiento del agua, la gestión de los residuos, la gestión de energía, en la agricultura para el abastecimiento de alimentos, entre muchos aspectos más (Mayor, 2014).

A la CyT le incumben aportar conocimientos para reducir la pobreza, hacer apta a la sociedad para prevenir desastres, reaccionar ante las catástrofes y emprender las tareas de reconstrucción, como también, acortar la brecha cognitiva y promover la cooperación intercultural, para un progreso seguro en el plano social y económico, para lograr los Objetivos del Desarrollo Sustentable (ODS) propuestos por la Organización de las Naciones Unidas (ONU) de conformidad con lo acordado en la Cumbre de Rio +20 (Cantú Martínez, 2015a; Cantú Martínez, 2016). Esta nueva orientación conlleva la consideración de la dimensión ecológica, con el propósito de hacer sostenible el desarrollo económico y social en relación con su sustento físico y material, [...] [con la finalidad de] hacer viable y duradero en el tiempo el bienestar humano, además de generalizable al conjunto de la población mundial en potencia (Toro, 2007, p. 153)

Para lograr esto, actualmente se advierte en la investigación tecnocientífica uno de los principales baluartes para el progreso de las sociedades contemporáneas, constituyéndose en el principal material de insumo para el impulso del desarrollo económico y social. Donde aunado a la 102

Pedro César Cantú-Martínez

(c) (i) \$)

Revista Economía y Sociedad by Universidad Nacional is licensed under a CreativeCommons Reconocimiento-NoComercial- 
innovación se ha constituido en el medio para transitar hacia la transformación de todas las sociedades. El presente documento tiene como fin brindar un aporte que examina la CyT para el desarrollo integral sustentable de la sociedad, como también esboza los desafíos que esto plantea en un marco regional para América Latina y el Caribe (ALyC).

\section{Metodología}

Se llevó a cabo una revisión de carácter documental que nos permitió acceder a las investigaciones hechas con anterioridad y circunscribir el objeto de estudio. La metodología utilizada residió en ta-localizar y recuperardocumentos de fuentes primarias como informes oficiales regionales y además, de fuentes secundarias que conllevan la valoración crítica de la información, como las bases de datos Scielo, Dialnet, Biblat, Directory Open Access Journals, Ebsco-host Fuente Académica y Web of Science-Social Sciences Citation Index, también en los metabuscadores de Google Académico y en los repositorios de Researchgate.net y Red de Bibliotecas Virtuales del Consejo Latinoamericano de Ciencias Sociales (CLACSO). Se definieron las búsquedas con palabras claves como ciencia, tecnología, indicadores, América Latina y Latinoamérica. Para posteriormente llevar a cabo una examinación descriptiva que nos permitió elegir las fuentes documentales con el objetivo de contribuir en una deliberación sobre las implicaciones de la CyT en el marco de un desarrollo perdurable para ALyC, lo que deja entrever los retos y avances que esto conlleva en el marco regional.

Esta indagación bibliográfica se realizó al seguir el rasgo de la CyT y su relación con el desarrollo sustentable, como también lo que representa para el progreso o retroceso de las sociedades contemporáneas, con lo cual nos permitió desarrollar el manuscrito y así concatenar las esferas de interés que yacen en el ámbito del tópico que estamos atendiendo para ALyC. Se estima que la información recabada y estructurada bajo el marco metodológico, mencionado con antelación, nos permitió configurarla unitariamente como también desplegar con ello una postura general, cuyo contenido sabemos, está en constante avance y pretende aproximarse a la realidad en que subsistimos como región.

\section{Marco conceptual}

\section{CyT en la cotidianidad}

De Angelis (2013) comenta,

Durante la Segunda Guerra Mundial tuvo lugar un suceso trascendental que marcaría un hito en las formas de relacionamiento de la ciencia con la sociedad: el surgimiento del Proyecto Manhattan y el posterior lanzamiento de las bombas atómicas sobre las ciudades de Hiroshima y Nagasaki. Este hecho transformaría la cosmovisión del 
hombre en el mundo, rompiendo los límites del conocimiento y marcando una nueva pauta en la relación de la política con la ciencia y la tecnología (p. 1).

Desde ese momento, la CyT retoman un papel importante, más allá del contexto castrense, observándose como una vía de carácter forzoso para los países, la cual deberían elegir para transitar a un escenario de oportunidades competitivas y con una escalada en el desarrollo económico. Fraga (1957) lo aseveraba al indicar que la invención en CyT

es uno de los elementos más poderosos de cambio social [...] el factor [científico y] tecnológico actúa, además, de un modo convérgete o multiplicador, al ser muchas las invenciones, cada una de las cuales actúa de diverso y acumulativo modo sobre un sistema de usos e instituciones sociales (p. 51).

De tal manera que Dagnino, Thomas y Davyt (1996) asientan que el "desarrollo tecnológico de posguerra de los países llamados avanzados se gestó a partir de la explotación de un stock de innovaciones mayores generadas desde mediados de la década del treinta hasta el fin de la Segunda Guerra Mundial" (p. 18) .

No es una sorpresa que los adelantos tecnocientíficos tengan una preponderancia en la esfera económica y social en que subsistimos, inclusive se puede señalar que determinan muchos aspectos del comportamiento y la forma de pensar sobre nuestras vidas: muestra la forma en que socializamos, la manera en que se actúa en el trabajo, en la forma de alimentarnos, en la manera de comunicarnos y cómo nos instruimos. Sin duda, estas transformaciones conllevan evidentes beneficios de carácter social, como lo señala Romo (2006):

1. A partir de la innovación en áreas como la agricultura, salud, información, transporte y energía es posible contribuir a reducir los niveles de pobreza e incrementar las capacidades humanas de la población.

2. Mediante un efecto indirecto, la ciencia y la tecnología también afectan positivamente el bienestar humano al estimular incrementos en la productividad y, con ella, el crecimiento económico y los niveles de ingresos. (p. 133)

En este sentido, la Organización para la Cooperación y el Desarrollo Económico (OCDE) ha mencionado que

los países que experimentaron aceleradas tasas de crecimiento en su productividad total de los factores entre la década de los ochenta y los noventa, también experimentaron tasas de crecimiento en el registro de patentes por arriba del promedio [en el ámbito de la ciencia y la tecnología]. Aun cuando las patentes no

104

Pedro César Cantú-Martínez

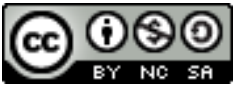

Revista Economía y Sociedad by Universidad Nacional is licensed under a CreativeCommons Reconocimiento-NoComercial- 
miden directamente la innovación, constituyen un indicador del desempeño tecnológico de un país. (Romo, 2006, p. 134)

En buena medida la investigación, la tecnología como la manera de innovar nos permiten afrontar los retos actuales que como sociedad tenemos que solucionar. Recordemos que los desafíos socioambientales son cada vez más complejos y suelen ser muy heterogéneos, como es el cambio climático, la producción energética mayormente limpia, contar con economías cada vez más competitivas, y satisfacer las necesidades de salud y alimentarias del conglomerado social que cada vez es mayor.

Existimos en un ambiente hondamente transfigurado por el conocimiento emanado de la CyT. Estamos conscientes de esto, al estar flanqueados a cada instante por los avances tecnológicos, tanto agrupadamente como de forma individual; por lo tanto, la "axiología de la ciencia se transmite a la axiología común. Las ciencias y tecnologías tienen, por tanto, una gran capacidad de provocar cambios sociales, puesto que afectan tanto a las maneras de hacer las cosas" (Menéndez \& Echeverría, 2014, p. 225).

Esto sucede paulatinamente, mediante lo que de forma habitual puntualizamos como generación de nuevos productos, que se ha denominado en la jerga científica como innovaciones. Jamison (2014) apunta que "muchas de las innovaciones tecnológicas importantes del primer período de industrialización fueron desarrolladas en contextos específicos para propósitos específicos, pero, a medida que la industrialización avanzaba, la producción y el proceso de la innovación se hicieron más sistemáticos y amplios"(p. 27).

Es pertinente diferenciar entre los términos semánticos de innovación, descubrimiento e invención, ya que, por lo general, a estos se les contempla como sinónimos, pero implican aspectos diferentes. De acuerdo con Bergeron y de la Torre (2015), sus connotaciones son “innovación es la aplicación de una idea conducida hasta su explotación efectiva por la sociedad. Descubrimiento es la puesta en evidencia de un fenómeno natural. Invención es un medio nuevo para lograr un objetivo" (p. 34).

Es así que innovación recuenta aquella transformación que induce cualquier novedad, o bien, de forma variada en un entorno, situación o producto en la cual el usuario final es el colectivo social; "y su éxito reposa en el número de adoptantes y en la importancia de las entidades (recursos, habilidades, etc.) que les son articulados" (Goulet \& Vinck, 2013, p. 15). Por este motivo la cultura de orden científico incorpora en nuestra sociedad cada vez más, innovaciones de todos aquellos artilugios que utilizamos en la vida cotidiana. Donde es indudable como CyT despliegan un predominio rotundo, en el desarrollo de todo ser humano. No obstante, esto, Menéndez \& Echeverría (2014) indican también que "el camino no va sólo de las tecnociencias a las vidas sociales, sino también de estas a aquellas" (p. 231). En esta dialéctica es perceptible observar: 
la creatividad, [que] es un proceso primario de ideación [...]. La innovación es un proceso secundario, ya aplicado, en donde la creatividad se aplica a la creación de un nuevo proceso, un producto o un servicio. El emprendimiento es un proceso [...] que lleva consigo poner en marcha [algo] [...] de la innovación ya desarrollada. A este conjunto de elementos se le ha denominado cultura emprendedora [en el ámbito social] (De la Fuente et. al., 2012, p. 946)

Esta cultura emprendedora crea conocimiento y se da aplicación al mismo, en un marco referencial en el cual se relacionan muchos "actores que no actúan unidireccionalmente sino a través de la generación de sinergias" (De Angelis, 2013, p. 1). En este proceder se observa -desde una perspectiva filosófica y particularmente sociológica- el "ethos clásico de la ciencia, propuesta originalmente por Robert Merton. El modelo mertoniano afirma que el científico comparte con sus colegas los resultados de su labor personal, razón por lo cual estos logros dejan de ser exclusiva propiedad individual" (Pruna, 1995, p. 214).

\section{Impacto social de la CyT}

CyT se han constituido en la sociedad en el dispositivo para sobreponerse y transformar el medio natural, como también el ámbito social y económico en el cual se desenvuelve todo ser humano. Por este motivo, se vincula a los avances tecnocientíficos con la procuración de una mejora sustancial en el bienestar y calidad de vida de las personas en un entorno afable. Sin embargo, es apreciable aún que estos beneficios no son igualmente permeados en el tejido social, donde lamentablemente persisten motivos de orden económico que favorecen a determinados grupos

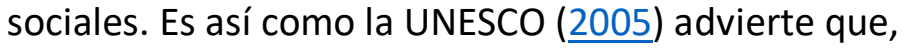

el conocimiento no se puede considerar una mercancía como las demás. La tendencia actual a la privatización e internacionalización de los sistemas de enseñanza superior merece una atención especial por parte de los encargados de adoptar decisiones y debería examinarse en el marco de un debate público, efectuando un verdadero trabajo de prospectiva a escala nacional, regional e internacional. El saber representa un bien común y su mercantilización merece, por consiguiente, un examen atento. ( $p$. 24)

Por otra parte, Sánchez (2009), comenta que las diferencias en la ejecución de avances científicos y tecnológicos en las naciones o entre los países, es producto de la parcelación inequitativa de los recursos económicos, de la distribución territorial que se hace y de la temporalidad en la que se lleva a cabo esta distribución de recursos financieros. Lo cual origina que los científicos, tecnólogos, académicos e intelectuales que se encuentran alejados de las fuentes de financiamiento no participen en situaciones de igualdad con aquellos pares que subsisten en las

106

Pedro César Cantú-Martínez

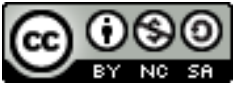

Revista Economía y Sociedad by Universidad Nacional is licensed under a CreativeCommons Reconocimiento-NoComercial- 
capitales centrales de los países, o bien de aquellos que radican en las naciones hegemónicas. Tünnerman, citado por Sánchez (2009), define tres aspectos que se desprenden de esta situación:

1) hay desigualdad en lo que respecta a los recursos financieros, instrumentales y técnicos para las investigaciones; 2) hay desigualdad en las oportunidades para la divulgación y aplicación de los resultados de los trabajos; 3) para asegurar la pertenencia a la academia mundial [o nacional], los miembros de los países periféricos dependientes aceptan restringir sus investigaciones a problemas, cuestiones, temas, métodos y técnicas definidos en los países centrales hegemónicos. (p. 30)

Al determinar que las prioridades del actuar científico observen el cumplimiento de expectativas de escenarios culturales disímiles a las locales. Que prescriben además que las pautas de la práctica científica, los modelos de certificación y la valoración de la labor científica sean básicamente establecidos en marcos de referencia externos.

Al hacer un balance sobre el actuar científico tecnológico subsisten resultados y consecuencias antagónicas. Donde la

responsabilidad por la destrucción de la riqueza natural y del detrimento de las condiciones sociales no es uniforme ni equitativa. Algunos son responsables directos por sus acciones intencionadas, otros responsables indirectos, la gran mayoría responsables por "el silencio cómplice", pero sin capacidad, ni poder, ni autoridad para tomar decisiones sobre el tema. (Mejía, 2013, p. 28)

Es así que todo cambio tecnocientífico que incide societariamente está también asociado a la génesis de múltiples problemas ambientales y está presente en las crisis de valores que emergen en nuestra sociedad. Parra y Cadena (2010, p. 338) comentan que los "hábitos de consumo, las formas de producción industrial, los métodos de explotación de recursos, el aumento de los desechos, entre otros, ejercen una presión tan grande sobre los ecosistemas que superan su resiliencia o capacidad de recuperación". Y en el contexto de la globalización se generalizan también los efectos de estas amenazas ambientales en el concierto internacional por la aplicación de la CyT.

La implementación de nuevo conocimiento y sus aplicaciones también se convierten en el origen de graves riesgos, lo que conlleva a que el empleo de nuevas tecnologías en algunas ocasiones produzca incertidumbre e inquietud en todo el colectivo social. Es así, que en el marco de la sustentabilidad emerge el principio de precaución o también denominado de cautela, el cual consiste en que si una acción o actividad se genera en un escenario de perplejidad científica es preferible no llevarla a cabo, y si esta se resuelve realizarla debe subsistir una profunda indagación sobre las consecuencias posibles para decretar las acciones pertinentes antes de su ejecución y 
con ello, abatir cualquier peligro, tanto para los seres humanos como para el entorno natural. Esto supone, sin lugar a duda, un cambio en la forma de valoración e implementación de todas las innovaciones tecnológicas y científicas (De Cózar, 2005).

En concordancia con lo anterior, se da paso a las dos vertientes que sobre sustentabilidad subsisten la fuerte y la débil. Para esto, López (2012) comenta las principales diferencias que entre ellas subsisten son:

1. [La sustentabilidad débil] se asienta en la creencia de que el agotamiento de determinados recursos naturales puede ser compensado por cambios tecnológicos o por la utilización de otros recursos o por capital económico. (p. 39)

2. [La sustentabilidad fuerte establece que] los recursos renovables o sustituibles pueden agotarse con la misma facilidad que los no renovables, y que el capital manufacturado no siempre puede ser sustituido por capital natural [...] la capacidad de sustitución entre los distintos tipos de capital está seriamente limitada por las características propias medioambientales, o la irreversibilidad de ciertos procesos naturales, [y] la incertidumbre sobre la evolución de los ecosistemas. (p. 41)

No obstante, estas dos posturas con respecto a la sustentabilidad, la aplicación de la CyT debe estar vinculada al uso racional de los insumos naturales, indistintamente de la posición que se favorezca. En ambas se debe tener en cuenta la "sustentabilidad ecológica del desarrollo [que] se refiere a la base física del proceso de crecimiento y objetiva la conservación de la dotación de recursos naturales incorporada a las actividades productivas" como comenta Guimarães (2002, p. 75). Pero, además, se considera importantemente la sustentabilidad ambiental que el mismo Guimarães expone que se "refiere a la relación con la manutención de la capacidad de carga de los ecosistemas, es decir, a la capacidad de la naturaleza para absorber y recomponerse de las agresiones antrópicas" (p. 75).

Al considerar lo anterior, debemos admitir que el progreso en CyT ha aportado ventajas al bienestar de todo colectivo social. Por tanto, el curso del progreso en el conocimiento científico y su aplicación social constituyen, hoy en día, insumos fundamentales para formar y reposicionar un proyecto de desarrollo económico, y se torna pertinente erigir, acompasadamente, un modelo que integre directrices incluyentes de carácter social, como también es conveniente que se acompañe de políticas relativas a los ámbitos sociales, productivos, ambientales y otras más que se contemplen beneficiosas, con la finalidad de promover el desarrollo sustentable, primeramente de manera local (Casas, 2015; Cantú-Martínez, 2015a). Aquí tomamos lo argumentado por Lara (2009) al indicar que:

Nuestra concepción de la ciencia y la tecnología trasciende el enfoque positivista o pragmático, que pretende calificar dichas operaciones por sus efectos utilitarios. Por

108

Pedro César Cantú-Martínez

(c) (i) \$)

Revista Economía y Sociedad by Universidad Nacional is licensed under a CreativeCommons Reconocimiento-NoComercial- 
el contrario, concebimos a la ciencia y la tecnología como expresiones de un proceso complejo del ser humano, en cuanto a la significación y transformación que producen en su entorno; representan, por tanto, un acto sociocultural (p. 17).

En este marco referencial la CyT, constituyen un medio para el ser humano mediante el cual los conocimientos se hacen tangibles y además estos se instituyen en "las formas racionales con que nos relacionamos con la realidad, asignando a ésta significados (ámbito referencial, genérico) y sentido (ámbito lógico-categorial, específico)" (Campos, 2004, pp. 8-9).

\section{Hallazgos para América Latina y el Caribe}

A continuación, se expone el escenario y los elementos encontrados en nuestra revisión, que aunamos de forma sucinta en una exposición de los indicadores tecnocientíficos para el desarrollo, como el papel de la CyT para el desarrollo social, se expone además las orientaciones para un desarrollo sustentable y finalmente los retos que ALyC afronta en CyT.

\section{Indicadores en ciencia y desarrollo (I+D)}

De acuerdo con la Red Iberoamericana de Indicadores de Ciencia y Tecnología (RICYT) en 2017, que tipifica el comportamiento de los indicadores en CyT de todas las naciones de América, junto con los países de España y Portugal, estipula en materia de inversión en I+D que

Brasil es el país iberoamericano que más esfuerzo relativo realiza en I+D, invirtiendo el $1,27 \%$ de su PBI en estas actividades. Portugal y España alcanzan el 1,24\% y 1,22\% respectivamente. El resto de los países latinoamericanos invirtieron menos del $0,7 \%$ de sus productos en I+D. Comparativamente, la inversión de los países de ALC e Iberoamérica continúa teniendo una baja intensidad en comparación a la de los países industrializados. Por ejemplo, Corea e Israel superan el 4\%, mientras que Alemania y EE. UU. rondan el 2,8\%. (RICYT, 2017, p. 14)

Esta misma instancia RICYT (2017) continúa estipulando que la inversión en dólares que ha realizado ALyC de manera conjunta en I+D constituyó el $3.5 \%$ de la cantidad total que en el mundo se ha realizado en este rubro. Sin embargo, este esfuerzo económico es muy pequeño frente a las naciones asiáticas cuya aplicación económica alcanzó el 41.4 \% de la inversión mundial hecha. Al desglosar esta inversión en I+D que se ha realizado, se denota que tres naciones en ALyC concentran este esfuerzo como son: Brasil (64\%), le siguen México y Argentina con el $17 \%$ y $7 \%$ respectivamente.

Por otra parte, en relación con personas dedicadas a la investigación se advierte que en 2015 ALyC contribuyó mundialmente con $3.9 \%$, número que es inferior a las contribuciones realizadas 
por el bloque asiático y europeo que aportaron el $42.3 \%$ y $30.9 \%$, mientras Estados Unidos y Canadá participaron con el $19 \%$. No obstante, estas cifras, encontramos a ALyC por encima de África y Oceanía que contribuyen con el $2.2 \%$ y $1.7 \%$ comparativamente (RICYT, 2017). Si pormenorizamos esta aportación de investigadores en AlyC, sobresale Brasil con 185853 investigadores, le sigue Argentina con 52970 y México con 29 921, lo que representa que la contribución brasileña sea casi cuatro y seis veces mayor que la participación argentina y mexicana.

En el marco de las patentes, este indicador refleja la inquietud de resguardar la propiedad intelectual y a su vez se relaciona con las capacidades de innovación del conocimiento con el que cuentan las naciones, y denota según datos de la Oficina de Patentes y Marcas de los Estados Unidos para países del resto del mundo que ALyC cuenta con 1.2 patentes por millón de habitantes en el lapso del año 2012 al 2015, inferior al de las primeras tres naciones en el mundo que son Estados Unidos, Japón e Israel, que cuentan con una tasa de 469.2, 427.1 y 403.5 respectivamente. No obstante, este escenario en ALyC las naciones con más patentes concedidas por millón de habitantes fueron Costa Rica, Chile y Uruguay con 3.4, 3.4 y 2.4 correspondientemente para el periodo de 2012-2015 (Comisión Económica para América Latina y el Caribe, 2016).

Definitivamente, en este breve análisis se puede reconocer que se ha buscado un cambio en materia I+D en la región ALyC, sin embargo, es evidente que este esfuerzo aún es infructuoso, porque es palpable todavía observar la heterogeneidad social y la segmentación económica subsistente (Rodríguez y Alvarado, 2008). Lo anterior, aunado a la estrechez económica prevaleciente en las naciones, restringe el apoyo a la incentivación de la I+D, situación que se torna apremiante porque se erige paralelamente también un proceso rápido de obsolescencia de la infraestructura y de las capacidades endógenas en materia de CyT.

\section{Investigación e innovación científica para el desarrollo social}

Tras el análisis de la documentación, actualmente se erige en la opinión pública, que el conocimiento científico unido a la innovación tecnológica, se instituyen como los principales patrimonios con los que cuenta la sociedad para impulsar el desarrollo sustentable y sufragar los rezagos sociales que subsisten. En este sentido, debemos retomar el contexto de desarrollo sustentable como aquel que cita Cantú Martínez (2015b),

entendemos el desarrollo sustentable, particularmente fuerte, como la vía para mejorar sostenidamente y de forma equitativa la calidad de vida del ser humano, edificado este desarrollo sustentable a partir de pautas apropiadas de protección, preservación y conservación del ambiente, con una explotación razonable de los

110

Pedro César Cantú-Martínez

$$
\text { (c) (i) (2) }
$$

Revista Economía y Sociedad by Universidad Nacional is licensed under a CreativeCommons Reconocimiento-NoComercial- 
recursos endógenos, de forma tal que se garanticen las expectativas y los intereses de las generaciones futuras, como también los de las actuales (p. 11).

Con esta línea conductora, la Organización de Estados Iberoamericanos para la Educación, la Ciencia y la Cultura (OEI) (2012) señala que la ciencia, tecnología e innovación se constituyen en instrumentos para la transformación, avance y sostén de las estructuras productivas, del uso cada vez más equilibrado de los recursos naturales, de los grandes alcances en materia de atención en salud hoy existentes, del progreso en el abasto de alimentos a comunidades vulnerables y del ascenso en el contexto educativo en muchas naciones, entre otros aspectos. Por lo tanto, en ALyC este ascenso es ostensible en "Brasil, México y Argentina, aunque con grandes diferencias entre ellos. Entre los restantes destaca Chile que ha experimentado un sostenido avance [...] Colombia y Venezuela también tienen un desarrollo mediano, en tanto que Cuba es muy exitosa en varias áreas" (OEI, 2012, p. 33).

En este sentido, destaca la forma transcendental y la vinculación de las actividades que realizan instituciones de la CyT con las demandas que emergen de las colectividades sociales, lo que enlaza los intereses sociales a los de orden científico y finalmente, con las condiciones productivas; esto ha transformado las estructuras sociales, productivas y científicas hasta ahora concebidas, al constituir a las políticas públicas en una elucidación del acontecer en materia de CyT (Muller, $\underline{2000)}$.

Por lo cual, se demanda un número mayor de profesionales competentes en los ámbitos de CyT, en condiciones de generar conocimiento de frontera, que innoven y sean capaces de adjudicárselo, adecuarlo y trasladarlo a la sociedad para conferir soluciones eficientes y cuyos beneficios se extienda a un mayor número de personas en el mundo. Por qué ha de considerarse que:

Sin entrar en cuestionamientos sobre el concepto de desarrollo, es evidente que los niveles de desarrollo entre las naciones se expresan, además de los aspectos socioeconómicos, a través de las diferencias en la producción de investigación científica y tecnológica [...] Un ejemplo de ello, lo encontramos en los adelantos científicostecnológicos en el ámbito de la salud, muchos de los cuales podrían permitir a los países subdesarrollados enfrentar un sinnúmero de problemas sanitarios locales, sin embargo, el alto costo que implica la importación de estos avances restringe su acceso y utilización (Vidales, 2009, p.37).

Esta alusión, describe el contexto regional en ALyC donde los progresos en materia de producción de alimentos, vivienda, medios para corregir el daño al medio ambiente, mejora de las condiciones de empleo y salud, aprovechamiento de los recursos naturales, por citar algunos, requieren comúnmente ser obtenidos del exterior y de naciones consideradas como proveedoras de avances en CyT (Vidales, 2009). A este escenario, apunta De Angelis (2013, p. 2) "es necesario 
abandonar la adaptación de modelos foráneos y afrontar el debate en torno a la necesidad de generar modelos, competencias, parámetros de calidad y orientaciones propias desde elementos idiosincrásicos a las características del desarrollo de los países latinoamericanos".

La forma de cristalizar lo anterior sería mediante la colaboración -hacia el interior de los países en ALyC- de las instancias gubernamentales, el sector empresarial, la sociedad civil y de los propios ciudadanos, que conllevaría una ejemplar interacción, que dentro de su virtuosidad permitiría una mayor igualdad social, participación política y un desarrollo más democrático, preceptos que nos encaminan a un progreso incluyente que está señalado en la Agenda 2030 y que se tipifica en los 17 ODS, al marcar que se tomará en cuenta "las diferentes realidades, capacidades y niveles de desarrollo de cada uno y respetando sus políticas y prioridades nacionales" (ONU, 2015, p. 4).

\section{Orientación para un desarrollo integral sustentable en el marco de la CyT}

Debe admitirse, que en la región se conjugan distintas perspectivas teóricas que pueden dar respuesta al escenario económico. Sin embargo, lo analizaremos desde el contexto de la perspectiva de la modernización. Históricamente en ALyC se ha subsistido en un contexto económico muy heterogéneo con relevantes reticencias que ha promovido el subdesarrollo y presencia principalmente de pobreza social. Es así como, de acuerdo con Pamela Cox (2006, p. xi) América Latina y el Caribe "se ha caracterizado por dos decepciones: un crecimiento débil y una pobreza y desigualdad persistentes".

Este entorno tipificado por Cox, primordialmente robustece el apropiamiento de los recursos del ambiente; la competitividad se funda en buscar un bajo costo de mano de obra, subsiste una alta concentración económica, además se hace presente la exclusión social, conjuntamente se incrementa la desigualdad social en distintos ámbitos como el educacional, el salarial, entre otros, por ende, los niveles de productividad son bajos hacia el interior de las naciones y el progreso socioeconómico mengua por el debilitamiento institucional (Vargas, 2008; Provencio, 2012).

Por su parte Provencio (2012) menciona que en

sociedades más igualitarias e integradas tienden a ser más productivas, a estimular una competitividad genuina que no esté basada en bajos salarios, a ser más estable a largo plazo; optimiza las capacidades creadas para concretarlas en la productividad, reduce costos colectivos asociados a la inseguridad pública, a las enfermedades y otros (p. 117).

Por ello ahora, estas economías están transitando a otra que requiere cada vez mayor soporte en el conocimiento, en la que el valor adicional radica en el valor agregado del producto o servicio 112

Pedro César Cantú-Martínez

(c) (†) $\$ 2$

Revista Economía y Sociedad by Universidad Nacional is licensed under a CreativeCommons Reconocimiento-NoComercial- 
que deriva de la innovación. Por lo tanto, es necesario reflexionar que las brechas que subsisten en temas como desarrollo social y económico, subyacen aspectos relacionados con la transferencia e innovación de la CyT. Recordemos como indica Albornoz (2015) el:

conocimiento científico, como expresión del espíritu humano e insumo necesario para el desarrollo económico y social, constituye una de las principales riquezas de las sociedades contemporáneas. La prosperidad de los países ha quedado asociada en gran medida al valor que agrega el conocimiento a la producción de bienes y servicios (p. 13).

De acuerdo con Martínez (2009), la ciencia se constituye en el fragmento más sobresaliente de la cultura, con el cual la sociedad puede detallar el entorno que le circunda; inclusive la considera una prerrogativa exclusiva de la especie humana. Es así, que estimular la competitividad hoy demanda un nuevo bosquejo socioeconómico respaldado en el conocimiento, cuyos puntales son la CyT (Cañedo, 2001). Este nuevo esquema favorece la comunicación estrecha entre el sector social, empresarial y los grupos de investigación tecnocientífica enclavados en las instituciones de educación superior (IES).

De tal forma, que sea preeminente el conocimiento en CyT, con la finalidad de que se inscriba en la conciencia social y se vea reflejado, este cambio, en la elaboración de agendas públicas, o bien, incorporándose en la restructuración de un nuevo contrato social de convivencia que influya positivamente en los aspectos de carácter educativo, de la vida cotidiana y del contexto cultural. Advertimos lo anterior, porque la CyT, a través de la innovación, conllevan en su génesis como aplicación, riesgos que dan pauta a la creación de nuevas amenazas, algunas de carácter global como el cambio climático, otras que se observan en la pérdida de empleos por la automatización de los procesos productivos. A esto se le conoce como "destrucción creadora" (Sebastián y Benavides, 2008; Fisher, 2010).

Por lo tanto, el conocimiento tecnocientífico es una actividad que emana de las propias colectividades sociales, el cual se edifica a partir de las competencias técnicas, valores y cultura que estas poseen (OEI, 2012), ya que como menciona Cantú Martínez (2012a, p. 16) "los conocimientos pueden ser de diferentes tipos como el cotidiano-empírico y científico". Y ahora este último se establece como un constituyente de carácter medular, tanto para el progreso económico como social, que influye en las condiciones de vida de las personas.

Cabe resaltar, no es suficiente el respaldar y promover políticas de incentivación en investigación básica, de manera que automáticamente se produzcan las mejoras y los avances en materia social, para esto es necesario que se fortalezca la relación educación-conocimiento-innovacióntransferencia, con el fin de robustecer las capacidades de la sociedad, pero, particularmente, el capital humano. Esto se observará mediante la generación de nuevos espacios de investigación, 
IES y parques industriales, entre otros aspectos (Cantú Martínez, 2012b), que den cuenta de la relación del nuevo conocimiento y las mejoras concretas en el ámbito social.

Lo anterior, demostrará los rasgos de los resultados de un programa de CyT encaminado al fortalecimiento de la cohesión social, difusión y divulgación del conocimiento, como también del desarrollo y sustentabilidad de la sociedad. Con lo cual, se podrá disminuir las trayectorias actuales de insustentabilidad y de desigualdad social, que aún subsisten en ámbitos como el empleo, la educación, ła-salud y alimentación, lo que demuestra la carencia de una cultura científica, que está privando a un gran número de personas de los beneficios de su conocimiento tecnocientífico. Arana (2005) avala lo antes citado al señalar,

personas bien informadas y conscientes sobre lo que es la ciencia y la tecnología, son la mejor base para una sociedad democrática, por lo tanto, los ciudadanos tienen que tomar conciencia de los logros de la ciencia y la tecnología, de su sentido humanista, de su poder y alcances. (p. 296)

Esto nos coloca en la necesidad de una renegociación de los vínculos existentes entre la ciencia y la sociedad, como lo indicaba López (1999), ya que se advierte es un problema complicado donde se involucran particularidades de orden económico, social, educativo, político y ético; todo ello promovido por la relevancia que ha logrado la CyT en el orbe.

\section{Desafíos de la CyT}

Los retos en ALyC para el desarrollo y cohesión social desde la perspectiva de la CyT, se yerguen en medio de una crisis de carácter económico, junto con un cuestionable proceder de la sociedad y la precepción que esta tiene con respecto a la CyT. Esto suscita un sistema social que carece de una convivencia humana, conveniente y decorosa (Pelechano, 2014). Que se distingue por el débil impulso de la sociedad hacia la competitividad y que muestra rasgos de inequidad distributiva, carencia de participación ciudadana, como de una disminuida cobertura de educación y atención en salud de las personas. Gran parte de este escenario es el resultado de las decisiones de las estructuras gubernamentales, que promovió

modos de hacer ciencia pensados en países con capacidades muy distantes de la realidad de América Latina [que] fueron implementados y adaptados acríticamente a partir de su presentación desde una concepción de la ciencia como pura, neutral y apolítica, generando incompatibilidades e incongruencias en los estudios y propuestas de los hacedores de política científica de la región y construyendo marcos perjudiciales para los diagnósticos y para las estrategias de acción adoptadas por los distintos países. (De Angelis, 2013, p. 2)

114

Pedro César Cantú-Martínez

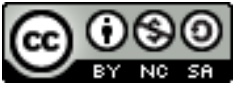

Revista Economía y Sociedad by Universidad Nacional is licensed under a CreativeCommons Reconocimiento-NoComercial- 
Se acepta por la sociedad que el rol, común, de la CyT es solo servir de forma instrumental a ella, sin embargo, esto hace a la tecnociencia indeterminada en su actuación social. Recordemos que la CyT pueden representar un notable papel en la función colectiva, desde una perspectiva no instrumental ni utilitarista, posicionándolas en una actuación que liberta y enriquece a cada miembro de la sociedad, lo que favorece el principio de justicia social equitativa e igualitaria.

Al hacer esta reflexión, a partir de la situación presente en ALyC, consideramos que los problemas que nos aquejan de forma social, económica y ambiental solo podrán ser franqueados cuando la razón ocupe un sitio prevaleciente en la dimensión humana, particularmente en aquella que hace empleo de la CyT, que transija a redireccionar el ejercicio intelectual hacia la concreción de políticas públicas más humanas y suprahumanas. En este sentido, Gómez (2008) apunta que toda persona

debe responder no sólo aquí y ahora, frente a los que padecen problemas de pobreza y se encuentran excluidos de los beneficios de la globalización por un comercio injusto. Además, el individuo debe responder ante las generaciones futuras por las posibles alteraciones a las que ha expuesto la vida en el planeta (p. 176).

En esta línea Sánchez y Osorio (2017, p. 45), apoyados en el análisis de Muller (2000), sostienen que "cada política pública se inscribe en una intención que tiene el Estado por intervenir en un dominio de la sociedad: puede ser para frenar la evolución de un fenómeno, como para transformarlo o adaptarlo a un curso de acción determinada."

Esta última aseveración es reveladora porque "la ciencia se ha vuelto un asunto de Estado. Es parte influyente del orden social [...] [concede al Estado la atribución de] manipular a la opinión pública" (Kaplan, 2003, p. 197), por lo cual es cuestionable en muchas ocasiones los niveles y trayectorias de su autonomía, en especial, cuando está supeditada a intereses no muy claros u obscuros. En este marco la política y la estructura gubernamental, de acuerdo con Kaplan (2003), puede constituirse en un impedimento o bien en un inductor para el desarrollo científico y por ende del social. En el primer escenario cuando las instancias gubernamentales y sus políticas sobre la ciencia se erigen como obstáculos es totalmente inconcebible y muy lamentable, ya que una cualidad de la relación moderna entre la ciencia, la tecnología y la sociedad debe darse en un cartabón donde tanto la CуT "deben ser autónomas, deben liberarse del control político o religioso" (Mitcham, 1990, p. 13), cuando no cumpla con el propósito de conllevar un progreso integral de la sociedad y sus miembros. Por lo cual el conocimiento en CyT según González (2010), puede erigirse en

una forma de exclusión social porque tener o no conocimiento científico o tecnológico genera una brecha entre quien lo posee y el que no, diferencia que marca el estatus de un individuo en la sociedad. Poseer y controlar cierto tipo de conocimiento genera 
relaciones sociales desiguales. Por ello, el impacto del conocimiento en un individuo es social y también personal, va más allá de los límites de lo público y lo privado. ( $p$. 4)

Como se observa, los desafíos de la CyT cuentan con innumerables interacciones que implican aspectos de orden ético, vinculadas al orden social y económico prevaleciente. Que condicionan su aplicación a los valores y constructos cognitivos pues determinan la sociedad en cuestión. Por lo tanto, el quehacer de la CyT

no puede pensarse más como una actividad desligada de la ética, debe fundamentarse como una actividad social, un hacer moral; ciencia y moral en la misma línea, un conjunto indivisible, inseparable por sus móviles y sus consecuencias. La dicotomía que consideraba que la ciencia buscaba la verdad y la ética la bondad de las acciones humanas, ha quedado entrelazada en un discurso único que unifica el ser y el deber ser como un todo integrado e indivisible, pensar en ciencia [y tecnología] es pensar en la trascendencia del saber cognitivo y las consecuencias naturales y sociales de cada representación modelada del mundo. (Mejía, 2013, p. 29)

De esta forma se va erigiendo, además -en el seno de todo colectivo humano- un tema trascendental que involucra la responsabilidad social, tanto colectiva como de forma individual, que fija el comportamiento de una sociedad como también del uso de la CyT por esta misma (Cantú Martínez, 2013a, Cantú Martínez, 2013b). Este valioso contrapeso permite a una sociedad internalizar más en la resolución de los problemas económicos y demandas sociales que se suceden en su territorio, con la finalidad de dignificar y mejorar el bienestar de las personas, contrarrestando así los intereses unilaterales que pueden surgir de algunos grupos de poder. Por lo tanto, la "ciencia y la tecnología son bienes públicos que pueden ser utilizados para aumentar el bienestar social y para resolver una diversidad de problemas económicos, sociales, culturales, ambientales y de preservación de recursos" (Olivé, 2008, p. 38).

Las condiciones actuales en ALyC han sido el resultado de una política pública en I+D y una estructura socioeconómica "con baja agregación de valor y bajas capacidades tecnológicas [y científicas]" de acuerdo con Suárez y Erbes (2014, p. 110). El papel de la CyT en este escenario en lugar de cerrar las brechas- exacerba las diferencias entre sus miembros lo cual es característico de las naciones de $\mathrm{ALyC}$, cuyos beneficios quedan en unos cuantos segmentos de la población, y por otra parte mantienen marginada a la mayoría de la base social.

\section{Consideraciones finales}

Todo lo anterior mencionado tiene una alta consonancia con el escenario de una práctica fructífera de la tecnociencia, la cual se encuentra necesariamente en un reposicionamiento de 116

Pedro César Cantú-Martínez

(c) (i) \$)

Revista Economía y Sociedad by Universidad Nacional is licensed under a CreativeCommons Reconocimiento-NoComercial- 
carácter social, para incidir de manera idónea en la toma de decisiones y elaboración de las políticas públicas que incurren en la resolución de los principales problemas socioambientales que nos aquejan en ALYC, como es la falta de empleo, la carencia de una educación de calidad, la desvinculación entre las personas que promueve la segregación social, la carga de enfermedad evitable, la desnutrición y las complicaciones de salud que emergen en el binomio maternoinfantil, así como la mala distribución y aprovechamiento de los alimentos. Figueroa (2009) indica que

ha quedado demostrado que el desarrollo es una condición que no se obtiene con el simple devenir histórico que abraza un proceso natural de evolución, la realidad advierte que esta noción se ubica lejos de representar una vía objetiva. Dicha condición que, sin duda, involucra la adquisición de capacidad sistemática para crear progreso tecnológico, demanda de una activa y decidida participación estatal, donde los esfuerzos sean canalizados, entre otras cosas, a la creación y levantamiento de una infraestructura científico-tecnológica sólida (p. 9).

Esto retoma importancia tras la reunión de Río +20 en 2012, cuya aspiración es la manifestación social de una СуТ para la sustentabilidad, donde este nuevo escenario de la labor científica se encamine en suscitar la transición a la sustentabilidad y permita resolver los avatares que nos aquejan y nos posicionan en una crisis. En el caso particular de la región de ALyC es necesario promover "procesos de cambio estructural, a la búsqueda de innovaciones de mayor complejidad tecnológica [como científica] y a la generación de capacidades endógenas" (Suárez y Erbes, 2014, p. 112). Lo anterior toma relevancia particularmente por lo que Cantú Martínez (2017) indica

hasta hace unos años el desarrollo económico de un país estribaba en [...] acumular capital y trabajo únicamente mediante el acto de incrementar la productividad [...] hoy en día estos pensamientos se han ido abandonando paulatinamente al examinarse en las distintas naciones que la ventaja reside sustancialmente en la manera eficiente de utilizar los recursos con los que se cuenta, empleando el conocimiento [científico y tecnológico] (p. 8).

Ese conocimiento científico y tecnológico, que Molina (1999) bosqueja como

parte constitutiva del espíritu moderno y su confianza [reside] en el papel transformador de la humanidad, sus posibilidades de conocer la realidad y de concebir, sobre la base de la acción práctica sobre el medio, un futuro deseado [para todo ser humano] (p. 9).

Recapacitemos que la CyT enriquece y alienta las capacidades para mejorar a cualquier sociedad y se erigen también en componentes fundamentales para un desarrollo perpetuo. 
Finalmente, no existe nada de extraordinario en llegar al 2030, pero la confluencia al cumplimiento de los ODS que se establece para esa fecha, la hace una valiosa oportunidad que poseen las estructuras sociales así como las gubernamentales para tomar decisiones y seguir impulsando la plataforma científica y tecnológica con la que se cuenta, constituyéndose así en un preámbulo al establecimiento de un desarrollo perdurable en ALyC sobre la base tecnocientífica para transformar el curso actual y construir capacidades de innovación sociocultural. Porque de acuerdo con Rodríguez y Alvarado (2008)

América Latina y el Caribe es una de las regiones más ricas del mundo, por su entorno natural y por las culturas que forman parte de ella. Sin embargo, es también una de las regiones donde más personas se encuentran sumidas en la pobreza y no acceden a los frutos del progreso técnico de la sociedad a la que pertenecen. A pesar de los grandes logros que la región ha alcanzado en materia de crecimiento económico durante los últimos 50 años, y de las diferentes políticas económicas implementadas, todavía no es posible afirmar que los resultados de ese crecimiento estén beneficiando a las grandes mayorías (p. 17).

\section{Referencias}

Albornoz, M. (2015). Repensar el papel de la ciencia y la tecnología en Iberoamérica. Organización de Estados Iberoamericanos para la Educación, la Ciencia y la Cultura (Primera edición, 13-30). Ciudad Autónoma de Buenos Aires: Organización de Estados Iberoamericanos para la Educación, la Ciencia y la Cultura. Recuperado de https://www.oei.es/historico/cienciayuniversidad/spip.php?article6025

Arana, M. H. (2005). La educación científico-tecnológica desde los estudios de la ciencia, tecnología, sociedad e innovación. Revista Tabula Rasa, 3, 293-313. Doi: https://doi.org/10.25058/20112742.239

Bergeron, M \& de la Torre, M. (2015). La pirámide de la innovación científica. Organización de Estados Iberoamericanos para la Educación, la Ciencia y la Cultura (pp. 31-40). Ciudad Autónoma de Buenos Aires: Organización de Estados Iberoamericanos para la Educación, la Ciencia y la Cultura la Recuperado de https://www.oei.es/historico/cienciayuniversidad/spip.php?article6025

Campos, M. A. (2004). Una aproximación sociocultural a los procesos cognoscitivos en el contexto educativo. Revista Perfiles educativos, 104 (26), 7-32. Recuperado de http://www.scielo.org.mx/pdf/peredu/v26n104/v26n104a2.pdf

118

Pedro César Cantú-Martínez

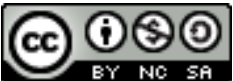

Revista Economía y Sociedad by Universidad Nacional is licensed under a CreativeCommons Reconocimiento-NoComercial- 
Cantú-Martínez, P. C. (2012a). Ciencia y conciencia humana. En P. Cantú Martínez (Ed.) Sustentabilidad científica: Introversión sobre la ciencia, conciencia y racionalidad social. (pp. 15-23). México: Clave Editorial Universidad Autónoma de Nuevo León. Recuperado de http://www.ameditores.com/libros-de-clave-editorial/sustentabilidad-cientifica

Cantú-Martínez, P. C. (2012b). Discurrir y apropiamiento de la ciencia por la sociedad. En P. Cantú Martínez (Ed.) Sustentabilidad científica: Introversión sobre la ciencia, conciencia y racionalidad social. (pp. 25-32). México: Clave Editorial Universidad Autónoma de Nuevo León. Recuperado de http://www.ameditores.com/libros-de-claveeditorial/sustentabilidad-cientifica

Cantú-Martínez, P. C. (2013a). Responsabilidad social empresarial en el marco de la sustentabilidad. Revista Economía y Sociedad, 44(18), 21-33. Recuperado de http://www.revistas.una.ac.cr/index.php/economia/article/view/5578

Cantú-Martínez, P. C. (2013b). Las instituciones de educación superior y la responsabilidad social en el marco de la sustentabilidad. Revista Electrónica Educare, 3(17), 41-55. Recuperado de http://www.scielo.sa.cr/pdf/ree/v17n3/a03v17n3.pdf

Cantú-Martínez, P. C. (2015a). Desarrollo sustentable. Antes y después de Río +20. México. Universidad Autónoma de Nuevo León y Organización Panamericana de la Salud. Recuperado de http://editorialuniversitaria.uanl.mx/index.php/2016/12/15/desarrollosustentable-antes-y-despues-de-rio20/

Cantú-Martínez, P. C. (2015b). Calidad de vida y sustentabilidad: Una nueva ciudadanía. Revista Ambiente y Desarrollo, 37(19), 9-22. Doi: https://doi.org/10.11144/Javeriana.ayd1937.cvsn

Cantú-Martínez, P. C. (2016). Los nuevos desafíos del desarrollo sustentable hacia 2030. Revista Ciencia UANL, 78(19), 27-32. Recuperado de http://eprints.uanl.mx/11001/

Cantú-Martínez, P. C. (2017). Economía del conocimiento para la sostenibilidad. Revista Economía y Sociedad, 51(22), 1-13. Doi: https://doi.org/10.15359/eys.22-51.5

Cañedo, R. (2001). Ciencia y tecnología en la sociedad: Perspectiva histórico-conceptual. Revista ACIMED, 1(9), 72-76. Recuperado de http://eprints.rclis.org/5204/1/aci051001.pdf 
Casas, R. (2015). Consideraciones para un nuevo enfoque latinoamericano sobre ciencia, tecnología e innovación para atender problemas sociales. En Organización de Estados Iberoamericanos para la Educación, la Ciencia y la Cultura (Ed.) Horizontes y desafíos estratégicos para la ciencia en Iberoamérica: Congreso Iberoamericano de Ciencia, Tecnología, Innovación y Educación. (pp. 83-95). Ciudad Autónoma de Buenos Aires: Organización de Estados Iberoamericanos para la Educación, la Ciencia y la Cultura. Recuperado de https://www.oei.es/historico/cienciayuniversidad/spip.php?article6025

Comisión Económica para América Latina y el Caribe (2016). Ciencia, tecnología e innovación en la economía digital. La situación de América Latina y el Caribe. Santiago: CEPAL. Recuperado de https://www.cepal.org/es/publicaciones/40530-ciencia-tecnologiainnovacion-la-economia-digital-la-situacion-america-latina

Cox, P. (2006). Prefacio. En: G. Perry, O. Arias, J. López, W. Maloney \& L. Servén. Reducción de la pobreza y crecimiento: círculos virtuosos y círculos viciosos. (pp. xi-xii). Bogotá. Banco Mundial y Mayol Ediciones S. A. Recuperado de http://siteresources.worldbank.org/INTLACINSPANISH/Resources/Reduccion_de_la_po breza.pdf

Dagnino, R., Thomas, H. \& Davyt, A. (1996). El pensamiento en ciencia, tecnología y sociedad en Latinoamérica: una interpretación política de su trayectoria. Revista Redes, 3(7), 13-51. Recuperado de https://ridaa.unq.edu.ar/handle/20.500.11807/504

De Angelis, I. (2013). Desafíos para el desarrollo en América Latina: La política científica y tecnológica en el siglo XXI Parte I. Revista Letras Internacionales, 168(7), 1-4. Recuperado de https://revistas.ort.edu.uy/letras-internacionales/article/view/427

De Cózar, J. M. (2005). Principio de precaución y medio ambiente. Revista Española de Salud Pública, 2(79), 133-144. Doi: https://doi.org/10.1590/S1135-57272005000200003

De la Fuente, J., Vera, M. M. \& Cardelle, M. (2012). Aportaciones de la psicología de la innovación y del emprendimiento a la educación, en la sociedad del conocimiento. Electronic Journal of Research in Educational Psychology, 28(10), 941-966. Doi: https://doi.org/10.25115/ejrep.v10i28.1544

Figueroa, S. A. (2009). El papel del Estado en el avance de la ciencia y tecnología, insumo vital en la construcción del desarrollo. En: S. Figueroa, G. Sánchez \& A. Vidales (Ed.) La ciencia y tecnología en el desarrollo. Una visión desde América Latina. (pp. 9-16). Zacatecas: Universidad Autónoma de Zacatecas. Recuperado de http://biblioteca.clacso.edu.ar/Mexico/uacp-uaz/20100322012242/CYTED.pdf

120

Pedro César Cantú-Martínez

(c) (†) $\$$ (O)

Revista Economía y Sociedad by Universidad Nacional is licensed under a CreativeCommons Reconocimiento-NoComercial- 
Fisher, E. (2010). La destrucción creadora y la teoría del valor trabajo: un modelo heterodoxo. Revista Reflexiones, 1(89), 97-103. Recuperado de https://revistas.ucr.ac.cr/index.php/reflexiones/article/view/11575/10920

Fraga, M. (1957). El impacto de la energía nuclear en la sociedad contemporánea. Revista de Estudios Políticos, 92, 51-66. Recuperado de https://dialnet.unirioja.es/servlet/articulo?codigo=2129074

Gómez, M.I. (2008). Las políticas públicas en la sociedad globalizada. Revista Opera, 8, 175-195. Recuperado de http://revistas.uexternado.edu.co/index.php/opera/article/view/770

González, J. A. (2010). El conocimiento como moneda de progreso. El concepto de distribución social del conocimiento en la Universidad Veracruzana: Un estudio de caso. (Tesis de Maestría). Recuperado de: https://cdigital.uv.mx/bitstream/123456789/41588/1/GonzalezHernandezJanette.pdf

Goulet, F. \& Vinck, D. (2013). La innovación por sustracción. Contribución a una sociología del desapego. Redes - Revista de Estudios Sociales de la Ciencia y la Tecnología, 19(36), 1349. Recuperado de http://publications.cirad.fr/une_notice.php?dk=573712

Guimarães, R. (2002). La ética de la sustentabilidad y la formulación de políticas de desarrollo. En: H. Alimonda (Ed.). Ecología política. Naturaleza, sociedad y utopía. (pp.53-82). Buenos Aires. CLACSO. Recuperado de http://bibliotecavirtual.clacso.org.ar/ar/libros/ecologia/ecologia.html

Jamison, A. (2014). Imaginación híbrida: una historia cultural de la ciencia y la tecnología. En: B. Laspra \& E. Muñoz (Ed.). Culturas científicas e innovadoras. Progreso social. (pp. 21-38). Buenos Aires: Editorial Universitaria de Buenos Aires. Recuperado de http://www.oei.es/historico/salactsi/culturascientificaseinnovadoras.php

Kaplan, M. T. (2003). La política científica: análisis y evaluación. En: J. Bokser (Ed.) Las ciencias sociales, universidad y sociedad. Temas para una agenda de posgrado. (pp. 193-204). México: Universidad Nacional Autónoma de México. Recuperado de https://books.google.com.mx/books?id=OPcNtshDbaYC\&printsec=frontcover\#v=onepa ge\&q\&f=false 
Lara, L. M. (2009). Acerca de la potencialidad secuestrada de la ciencia y tecnología. Por una idea del desarrollo multidimensional. En: S. Figueroa, G. Sánchez \& A. Vidales (Ed.) La ciencia y tecnología en el desarrollo. Una visión desde América Latina. (pp. 17-22). Zacatecas: Universidad Autónoma de Zacatecas. Recuperado de http://biblioteca.clacso.edu.ar/Mexico/uacp-uaz/20100322012242/CYTED.pdf

López, I. (2012). Sostenibilidad débil y fuerte y democracia deliberativa -el caso de la agenda 21 local de Madrid. (Tesis doctoral). Recuperado de https://earchivo.uc3m.es/bitstream/handle/10016/16270/tesis_lopez_pardo_2012.pdf

López, J. A. (1999). Los estudios de ciencia, tecnología y sociedad. Revista Iberoamericana de Educación, 20, 217-225. Recuperado de http://www.oei.es/historico/salactsi/cerezorie20.htm

Martínez, L. J. (2009). La ciencia y nosotros. Revista El Profesional de la Información, 5(18), 485492. Doi: https://doi.org/10.3145/epi.2009.sep.01

Mayor, F. (2014). Prólogo. Ciencia y tecnología como vigías en una sociedad en cambio. En: B. Laspra \& E. Muñoz (Ed.). Culturas científicas e innovadoras. Progreso social. (pp. 7-14). Buenos Aires: Editorial Universitaria de Buenos Aires. Recuperado de http://www.oei.es/historico/salactsi/culturascientificaseinnovadoras.php

Mejía, E. (2013). Bio-contabilidad: ética, ciencia y tecnología para la sostenibilidad. Revista Libre Empresa, 10(2), 13-33. Recuperado de https://dialnet.unirioja.es/servlet/articulo?codigo=6586833

Menéndez, A. \& Echeverría, J. (2014). Ciencia y tecnología en nuestras vidas: una visión axiológica. En: B. Laspra \& E. Muñoz (Ed.). Culturas científicas e innovadoras. Progreso social. (pp. 223-236). Buenos Aires: Editorial Universitaria de Buenos Aires. Recuperado de http://www.oei.es/historico/salactsi/culturascientificaseinnovadoras.php

Mitcham, C. (1990). En busca de una nueva relación entre ciencia, tecnología y sociedad. En: M. Medina \& J. Sanmartín. (Ed.) Ciencia, tecnología y sociedad: Estudios interdisciplinares en la universidad, en la educación y en la gestión política y social. ( pp. 11-19). Barcelona: Editorial Anthropos. Recuperado de https://books.google.com.mx/books?id=r23PqkSCHEC\&printsec=frontcover\#v=onepage \&q\&f=false

122 
Molina, A. (1999). La ciencia, una construcción humana. En: A. Molina. (Ed.) Ciencia, Tecnología y Sociedad. Selección de textos de quehacer científico I. (pp. 9-14). Santo Domingo, República Dominicana: Instituto Tecnológico de Santo Domingo. Recuperado de https://books.google.com.mx/books?id=K08nY8XdBCkC\&printsec=frontcover\&hl=es\#v $=$ onepage $\& q \& f=$ false

Muller, P. (2000). L'analyse cognitive des politiques publiques: vers une sociologie politique de l'action publique. Revue française de science politique, 2(50), 189-208. Doi: https://doi.org/10.3406/rfsp.2000.395464

Organización de las Naciones Unidas. (2015). Proyecto de documento final de la cumbre de las Naciones Unidas para la aprobación de la agenda para el desarrollo después de 2015. Recuperado de https://unctad.org/meetings/es/SessionalDocuments/ares70d1_es.pdf

Organización de Estados Iberoamericanos para la Educación, la Ciencia y la Cultura. (2012). Ciencia, tecnología e innovación para el desarrollo y la cohesión social. Madrid: Organización de Estados Iberoamericanos para la Educación, la Ciencia y la Cultura. Recuperado de https://www.oei.es/historico/cienciayuniversidad/?article5200

Olivé, L. (2008). La ciencia y la tecnología en la sociedad del conocimiento. Ética, política y epistemología. (Primera edición). México: Fondo de Cultura Económica. Recuperado de http://goo.gl/g8ssJc

Parra, A. \& Cadena, Z. (2010). El medio ambiente desde las relaciones de ciencia, tecnología y sociedad: un panorama general. Revista CS, 6, 331-359. Doi: https://doi.org/10.18046/recs.i6.471

Pelechano, V. (2014). Ciencia, sabiduría y sociedad. Análisis y modificación de conducta, 161162(40), 1-28. Doi: https://doi.org/10.33776/amc.v40i0.2611

Provencio, E. (2012). La estrategia distributiva para el crecimiento. En: R. Becerra. (Coord.) Equidad Social y parlamentarismo balance de treinta años. (8-130). México: Siglo XXI Editores e Instituto de Estudios para la Transición Democrática. Recuperado de https://www.gandhi.com.mx/equidad-social-y-parlamentarismo-balance-de-treinta-aos

Pruna, P. M. (1995). Ciencia: ethos y método. Revista de la Sociedad Española de Historia de las Ciencias y de las Técnicas, 34(18), 213-222. Recuperado de https://dialnet.unirioja.es/descarga/articulo/62147.pdf 
Red Iberoamericana de Indicadores de Ciencia y Tecnología. (2017). El Estado de la Ciencia. Principales Indicadores de Ciencia y Tecnología Iberoamericanos / Interamericanos, 2017. Buenos Aires: Red de Indicadores de Ciencia y Tecnología Iberoamericana e Interamericana. Recuperado de http://www.ricyt.org/files/Estado\%20de\%20la\%20Ciencia\%202017/El_Estado_de_la_C iencia_2017.pdf

Rodríguez, A. \& Alvarado, H. (2008). Claves de la innovación social en América Latina y el Caribe. (Primera edición). Santiago: Naciones Unidas. Recuperado de https://repositorio.cepal.org/bitstream/handle/11362/2536/1/S0800540_es.pdf

Romo, D. (2006). El impacto de la ciencia y la tecnología en el desarrollo de México. En: A. Borjas \& M. Bucio (Ed.) La ciencia y la tecnología como ejes de la competitividad de México. (pp.127-194). México: Centro de Estudios Sociales y de Opinión Pública. Recuperado de http://cort.as/-Hq5r

Sánchez, G. (2009). Ciencia - Tecnología - Desarrollo: Una relación cuestionada y en disputa. En: S. Figueroa, G. Sánchez \& A. Vidales (Ed.) La ciencia y tecnología en el desarrollo. Una visión desde América Latina. (pp. 23-35). Zacatecas: Universidad Autónoma de Zacatecas. Recuperado de http://biblioteca.clacso.edu.ar/Mexico/uacpuaz/20100322012242/CYTED.pdf

Sánchez, P. \& Osorio, L. (2017). Instrumentos de política científica, tecnológica y de innovación en América Latina. Principales tendencias en Argentina, Brasil y México. En: Red Iberoamericana de Indicadores de Ciencia y Tecnología (Ed.). El Estado de la Ciencia. Principales Indicadores de Ciencia y Tecnología Iberoamericanos / Interamericanos, 2017. (pp. 45-54). Buenos Aires: Red de Indicadores de Ciencia y Tecnología Iberoamericana e Interamericana. Recuperado de http://www.ricyt.org/files/Estado\%20de\%20la\%20Ciencia\%202017/El_Estado_de_la_C iencia_2017.pdf

Sebastián, J. \& Benavides, C. (2008). Ciencia, Tecnología y Desarrollo. (Primera edición). Madrid: Agencia Española de Cooperación Internacional. Recuperado de http://www.cervantesvirtual.com/obra/ciencia-tecnologia-y-desarrollo--0/

Suárez, D. \& Erbes, A. (2014). Desarrollo y subdesarrollo latinoamericano: un análisis crítico del enfoque de los sistemas de innovación para el desarrollo. Revista Redes, 38(20). 97-119. Recuperado de http://www.redalyc.org/articulo.oa?id=90745924003

124

Pedro César Cantú-Martínez

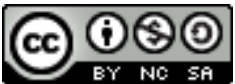

Revista Economía y Sociedad by Universidad Nacional is licensed under a CreativeCommons Reconocimiento-NoComercial- 
Toro, F. J. (2007). El desarrollo sostenible: un concepto de interés para la geografía. Revista Cuadernos Geográficos, 1(40), 149-181. Recuperado de http://www.ugr.es/ cuadgeo/docs/articulos/040/040-008.pdf

Organización de las Naciones Unidas para la Educación, la Ciencia y la Cultura. (2005). Hacia las sociedades del conocimiento. Francia: UNESCO. Recuperado de http://unesdoc.unesco.org/images/0014/001419/141908s.pdf

Vargas-Hernández, J. G. (2008). Análisis crítico de las teorías del desarrollo económico. Revista Economía, Gestión y Desarrollo, 6, 109-131. Recuperado de https://www.researchgate.net/publication/23935088_ANALISIS_CRITICO_DE_LAS_TEO RIAS_DEL_DESARROLLO_ECONOMICO

Vidales, A. (2009). Ciencia y tecnología para el desarrollo: el dilema de las políticas científicotecnológicas en países periféricos. En: S. Figueroa, G. Sánchez \& A. Vidales (Ed.) La ciencia y tecnología en el desarrollo. Una visión desde América Latina. (pp. 37-44). Zacatecas: Universidad Autónoma de Zacatecas. Recuperado de http://biblioteca.clacso.edu.ar/Mexico/uacp-uaz/20100322012242/CYTED.pdf 